\title{
ECONOMETRIC MODEL FOR ANAL YSING THE STRUCTURAL FUNDS ABSORPTION AT REGIONAL LEVEL - SECTORAL OPERATIONAL PROGRAMME HUMAN RESOURCES DEVELOPMENT
}

\author{
Oana Gherghinescu ${ }^{1}$
}

\begin{abstract}
An econometric model represents an important tool for simulating the principal mechanisms of economic systems. This could be applied at different scales, namely regional, national and international. When approaching this research field it should be kept in mind, permanently, that macroeconomic theory represents a dynamic environment, with a large diversity of (sub) theories, each of them claiming as being the most relevant. There is a large variety of such econometric models, but the basic principles of conceiving them are mostly the same. The present paper proposes an ARCH like model in order to analyse the absorption of structural funds within the Sectoral Operational Programme - Human Resources Management, at regional level. There are made considerations on the convergence of the model and on the applied statistical tests. There is also emphasized the role that such a model could play in improving future programming exercises.
\end{abstract}

Key words: Model Construction and Estimation, Model Evaluation and Testing, Regional Development Policy

JEL codes: C51, C52, R58.

\section{Introduction}

The Sectoral Operational Programme Human Resources Development (SOP HRD) was elaborated taking into account the Community provisions in this field, according to the Council Regulation (EC) No. 1083/2006 laying down general provisions on the European Regional Development Fund, the European Social Fund and the Cohesion Fund, the Regulation (EC) No. 1081/2006 of the European Parliament and of the Council on the European Social Fund, the Commission Regulation No. 1828/2006 setting out rules for the implementation of Council Regulation (EC) No. 1083/2006 laying down general provisions on the European Regional Development Fund, the European Social Fund and the Cohesion Fund.

The general objective of SOP HRD is the development of human capital and increasing competitiveness, by linking education and lifelong learning with the labour market and ensuring increased opportunities for future participation on a modern, flexible and inclusive labour market for 1,650,000 people.

The SOP HRD general objective may be split into a series of specific objectives:

$\circ$ promoting quality initial and continuous education and training, including higher education and research;

○ promoting an entrepreneurial culture and improving quality and productivity at work;

$\circ$ supporting entry or re-entry into the labour market of young people and the long term unemployed;

○ developing a modern, flexible, inclusive labour market;

○ promoting re-entry into the labour market of inactive people, including those in rural

\footnotetext{
${ }^{1}$ University of Craiova. oana.gherghinescu@ucvcentral.ro
} 
areas;

○ improving public employment services;

- facilitating access to education and the labour market of disadvantaged and vulnerable groups.

The Sectoral Operational Programme Human Resources Development is structured on 7 Priority Axes and 21 Key Areas of Intervention. These 7 Priority Axis are: Education and training in support for growth and development of knowledge based society, Linking life long learning and labour market, Increasing adaptability of workers and enterprises, Modernizing the public employment service, Promoting active employment measures, Promoting social inclusion and Technical assistance.

The principle of the sustainable development shall be considered integral to all objectives and Axis of the SOP HRD. The SOP HRD priorities have to be met whilst taking into account the protection and improvement of the environment. In particular, specific activities shall be implemented in order to support the development of SMEs in the sectors of environment protection, tourism and cultural services; to develop best practice for SMEs in relation to effective environmental management the adoption and use of pollution prevention technologies, integration of clean technologies to production; to promote publicity campaigns for encouraging the Corporate Social Responsibility of SMEs and for ensuring the effective participation of citizens in environmental protection and controlling pollution.

Projects of Education for Sustainable Development are central to the key aims of the EC strategy and are expected to receive full support from ESF. The general goal of such projects is to have young people better prepared to face the challenge of the present and of the future and to act responsibly for the next generations. To this end the initiatives to be taken must develop learning in all the fundamental areas, learning to know, learning to do, learning to be, learning to live together and learning to transform oneself and society.

The basic idea of the present model, due to the lack of consistent time-series for the structural funds absorption process, is to use a specific model with a mix input. This mix input takes into consideration data related to the pre-accession period and to the first monitoring exercise of structural funds absorption. Under this approach the time interval for the combined process raises from 3 to 10 years.

\section{Research Methodology and Paper Review}

Autoregressive Conditional Heteroskedasticity (ARCH) models are specifically designed to model and forecast conditional variances. The variance of the dependent variable is mod- eled as a function of past values of the dependent variable and independent, or exogenous variables.

ARCH models were introduced by Engle (1982) and generalized as GARCH (Generalized ARCH) by Bollerslev (1986). These models are widely used in various branches of econometrics, especially in financial time series analysis. See Bollerslev, Chou, and Kroner (1992) and Bollerslev, Engle, and Nelson (1994) for recent surveys.

In order to perform the Analysis we will use such a statistical model applied to the structural model presented in Fig. 1.

The following set of variables has been considered:

ABS_POS_DRU_P - time serie with payments in SOP-HRD

ABS_POS_DRU_V - time serie with contracted amounts in SOP-HRD

HRD_PRE_xx - time series for HRD pre-accession funds at regional level

INFRA_PRE_xx - time series for Infrastructure pre-accession funds at regional level

IRU $(\mathbf{x}, \mathbf{y})$

POP_REG

- HRD data at regional level

- population at regional level 
POS_DRU_P_X - payments within SOP-HRD at regional level

PO_DRU_V_X - contracted amounts within SOP-HRD at regional level

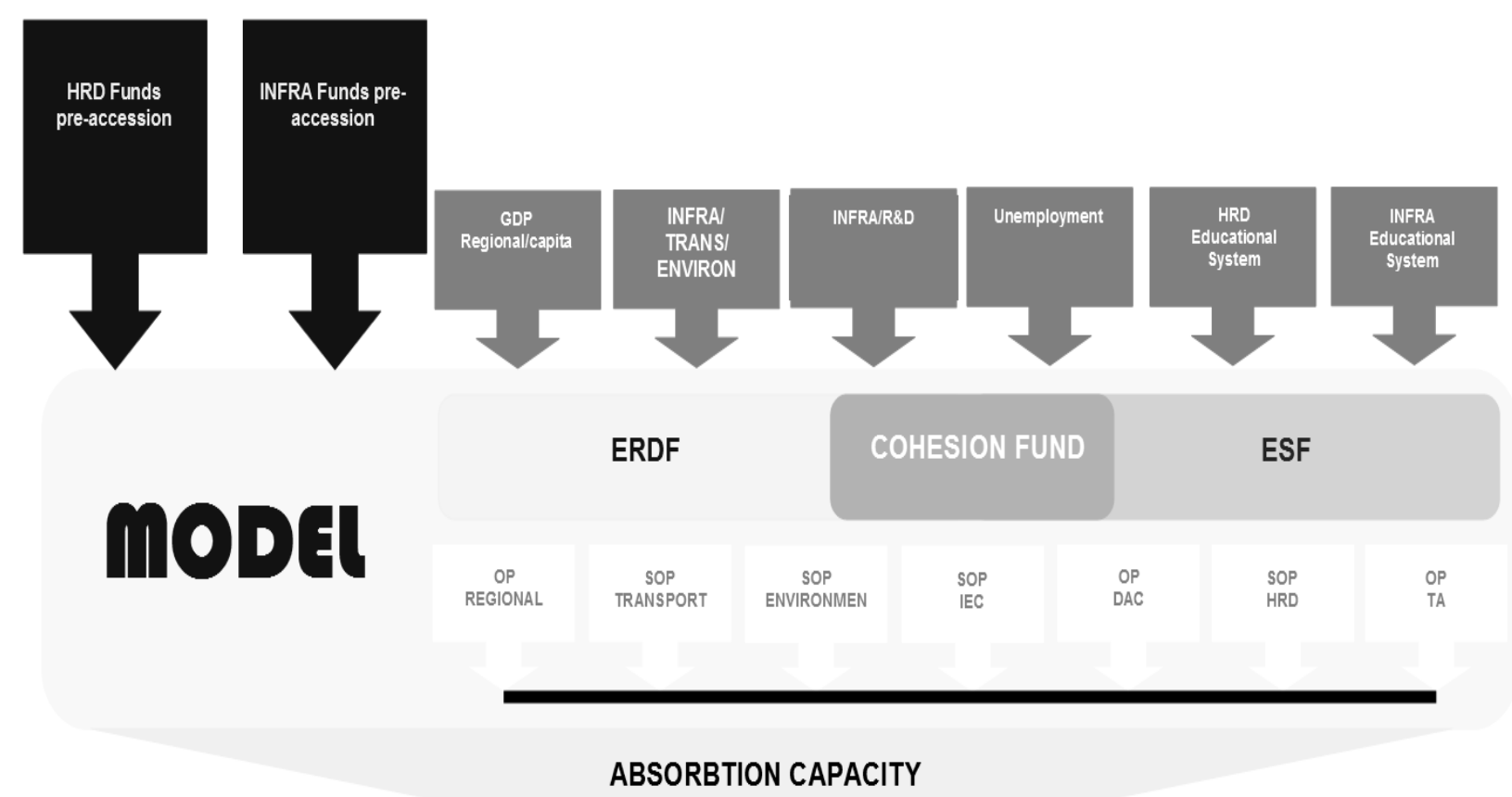

Fig. no. 1. The structural model and the position of the current analysis into it

\section{Results and Conclusions}

After running the model, next results have been obtained, in the case of all 8 development regions:

a) South Region

Dependent Variable: Implicit Equation Estimated by GMM

Method: ML - ARCH

Date: 09/11/10 Time: 20:44

Sample(adjusted): 27

Included observations: 6 after adjusting endpoints

Convergence achieved after 1 iterations

LOG(POS_DRU_P_X(1,1))-(C(1)*LOG(POS_DRU_V_X $(1,1)$

*POP_REG $(1,1) *$ PIB_REG $(1,1))+\mathrm{C}(2) *$ LOG(HRD_PRE_S(-1)

*POS_DRU_V_X $(1,1) * \operatorname{IRU}(1,1) * \operatorname{IRU}(1,2) * \operatorname{IRU}(1,3) * \operatorname{IRU}(1,4)$

$* \operatorname{IRU}(1,5) * \operatorname{IRU}(1,6) * \operatorname{IRU}(1,9) * \operatorname{IRU}(1,10) * \operatorname{IRU}(1,11) * \operatorname{IRU}(1,12))+\mathrm{C}(3)$

*LOG(SOMAJ_S(-1)*POS_DRU_V_X(1,1)*IRU $(1,1) * \operatorname{IRU}(1,2)$

*IRU(1,3)*IRU(1,4)*IRU(1,5)*IRU(1,6)*IRU(1,9)*IRU(1,10)*IRU(1,1

1)

*IRU(1,12)))

\begin{tabular}{rrrrr}
\hline \hline & Coefficient & Std. Error & z-Statistic & Prob. \\
\hline \hline $\mathrm{C}(1)$ & 0.398452 & 0.004379 & 90.98291 & 0.0000 \\
$\mathrm{C}(2)$ & $2.92 \mathrm{E}-11$ & $6.51 \mathrm{E}-05$ & $4.49 \mathrm{E}-07$ & 1.0000
\end{tabular}




\begin{tabular}{rrrrr}
\multicolumn{1}{c}{$\mathrm{C}(3)$} & $3.80 \mathrm{E}-11$ & $8.44 \mathrm{E}-05$ & $4.50 \mathrm{E}-07$ & 1.0000 \\
$\mathrm{C}(4)$ & $3.89 \mathrm{E}-24$ & $2.76 \mathrm{E}-05$ & $1.41 \mathrm{E}-19$ & 1.0000 \\
$\mathrm{C}(5)$ & 0.150000 & 12.05057 & 0.012448 & 0.9901 \\
$\mathrm{C}(6)$ & 0.600000 & 8.370669 & 0.071679 & 0.9429 \\
\hline \hline Akaike info & -48.59101 & Sum squared resid & $3.60 \mathrm{E}-23$ \\
criterion & & & \\
Schwarz criterion & -48.79925 & Log likelihood & 151.7730 \\
$\begin{array}{l}\text { Durbin-Watson } \\
\text { stat }\end{array}$ & 1.466228 & & \\
\hline \hline
\end{tabular}

Equation: DRU_S Workfile: DATE_TEZA

$\square \square$

\begin{tabular}{l|l|l|l|l|l|l|l|l|l|} 
View & Procs & Objects & Print & Name & Freeze & Estimate & Forecast & Stats & Resids \\
\hline
\end{tabular}

\section{Correlogram of Standardized Residuals}

Date: 09/12/10 Time: 00:05

Sample: 27

Included observations: 6

\begin{tabular}{|c|c|c|c|c|c|c|c|c|c|}
\hline \multicolumn{3}{|c|}{ Autocorrelation } & \multicolumn{3}{|c|}{ Partial Correlation } & $\mathrm{AC}$ & PAC & Q-Stat & Prob \\
\hline 1 & 다 & & 1 & 니 & 1 & $1-0.090$ & -0.090 & 0.0778 & 0.780 \\
\hline 1 & c & & 1 & c & 1 & $2-0.098$ & -0.107 & 0.1939 & 0.908 \\
\hline 1 & & & 1 & & 1 & $3-0.031$ & -0.052 & 0.2096 & 0.976 \\
\hline 1 & $\square$ & & 1 & & 1 & $4-0.401$ & -0.430 & 4.0747 & 0.396 \\
\hline
\end{tabular}

Fig. no. 2. Correlogram of Standardized Residuals

\begin{tabular}{|c|c|c|c|c|c|c|c|c|}
\hline \multicolumn{2}{|c|}{$\square$ Equation: DRU_S } & \multicolumn{5}{|c|}{ Workfile: DATE_TEZA } & \multicolumn{2}{|c|}{0 回 } \\
\hline View & \begin{tabular}{l|l|} 
Procs & Objects \\
\end{tabular} & \begin{tabular}{l|l} 
Print & Name \\
\end{tabular} & Freeze & Estimate & Forecast & Stats & Resids & \\
\hline obs & Actual & Fitted & Residual & & Resic & dual $F$ & Plot & \\
\hline obs & Actual & Fitted & Residual & & Resi & dual $P$ & Jot & $\overline{4}$ \\
\hline 2 & 0.00000 & $7.4 \mathrm{E}-13$ & $-7.4 \mathrm{E}-13$ & & & & & \\
\hline 3 & 0.00000 & $-2.7 \mathrm{E}-12$ & 2.7E-12 & & & & & $\equiv$ \\
\hline 4 & 0.00000 & $-3.5 \mathrm{E}-13$ & $3.5 \mathrm{E}-13$ & & & & & \\
\hline 5 & 0.00000 & $-1.9 \mathrm{E}-12$ & $1.9 \mathrm{E}-12$ & & & & & \\
\hline 6 & 0.00000 & $-7.6 \mathrm{E}-13$ & 7.6E-13 & & & & & \\
\hline 7 & 0.00000 & $4.9 \mathrm{E}-12$ & $-4.9 \mathrm{E}-12$ & $\begin{array}{lll}2 & 0-1 \\
\end{array}$ & & & & - \\
\hline & 1 & $\square$ & III & & & & & \\
\hline
\end{tabular}

Fig. no. 3. Data for Correlogram of Standardized Residuals

b) South_West Region

Dependent Variable: Implicit Equation Estimated by GMM

Method: ML - ARCH

Date: 09/11/10 Time: 20:34

Sample(adjusted): 27

Included observations: 6 after adjusting endpoints

Convergence achieved after 1 iterations 
Method: ML - ARCH

Date: 09/12/10 Time: 00:40

Sample(adjusted): 27

Included observations: 6 after adjusting endpoints

Convergence achieved after 1 iterations

LOG(POS_DRU_P_X(3,1))-(C(1)*LOG(POS_DRU_V_X(3,1)

*POP_REG(3,1)*PIB_REG(3,1))+C(2)*LOG(HRD_PRE_SE(-1)

*POS_DRU_V_X(3,1)*IRU $(3,1) * \operatorname{IRU}(3,2) * \operatorname{IRU}(1,3) * \operatorname{IRU}(3,4)$

$* \operatorname{IRU}(3,5) * \operatorname{IRU}(3,6) * \operatorname{IRU}(3,9) * \operatorname{IRU}(3,10) * \operatorname{IRU}(3,11) * \operatorname{IRU}(3,12))+\mathrm{C}(3)$

*LOG(SOMAJ_SE(-1)*POS_DRU_V_X(3,1)*IRU(3,1)*IRU(3,2)

$* \operatorname{IRU}(3,3) * \operatorname{IRU}(3,4) * \operatorname{IRU}(3,5) * \operatorname{IRU}(3,6) * \operatorname{IRU}(3,9) * \operatorname{IRU}(3,10) * \operatorname{IRU}(3,11)$ *IRU $(3,12)))$

\begin{tabular}{crrrr}
\hline \hline & Coefficient & Std. Error & z-Statistic & Prob. \\
\hline \hline $\mathrm{C}(1)$ & 0.404815 & 0.003868 & 104.6536 & 0.0000 \\
$\mathrm{C}(2)$ & $-1.37 \mathrm{E}-11$ & $8.26 \mathrm{E}-05$ & $-1.66 \mathrm{E}-07$ & 1.0000 \\
$\mathrm{C}(3)$ & $-6.65 \mathrm{E}-12$ & $8.25 \mathrm{E}-05$ & $-8.05 \mathrm{E}-08$ & 1.0000 \\
$\mathrm{C}(4)$ & $1.86 \mathrm{E}-24$ & $1.70 \mathrm{E}-05$ & $1.09 \mathrm{E}-19$ & 1.0000 \\
$\mathrm{C}(5)$ & 0.150000 & 9.683399 & 0.015490 & 0.9876 \\
$\mathrm{C}(6)$ & 0.600000 & 7.682661 & 0.078098 & 0.9378 \\
\hline \hline Akaike info criterion & -49.26419 & Sum squared resid & $1.72 \mathrm{E}-23$ \\
Schwarz criterion & -49.47243 & Log likelihood & 153.7926 \\
Durbin-Watson stat & 1.525282 & & & \\
\hline \hline
\end{tabular}

Equation: DRU_SE Workfile: DATE_TEZA

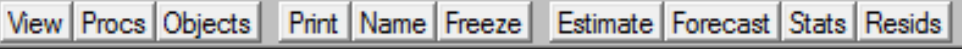

\section{Correlogram of Standardized Residuals}

Date: 09/12/10 Time: 00:41

Sample: 27

Included observations: 6

\begin{tabular}{|c|c|c|c|c|c|c|c|c|c|}
\hline \multicolumn{3}{|c|}{ Autocorrelation } & \multicolumn{3}{|c|}{ Partial Correlation } & $\mathrm{AC}$ & $\mathrm{PAC}$ & Q-Stat & Prob \\
\hline 1 & 나 & I & । & 다 & । & $1-0.083$ & -0.083 & 0.0668 & 0.796 \\
\hline । & 4 & I & 1 & 나 & 1 & $2-0.101$ & -0.109 & 0.1888 & 0.910 \\
\hline 1 & $\square$ & 1 & 1 & $\square$ & 1 & $3-0.206$ & -0.229 & 0.8708 & 0.832 \\
\hline 1 & $\square$ & 1 & 1 & & 1 & $4-0.349$ & -0.436 & 3.7923 & 0.435 \\
\hline
\end{tabular}

Fig. no. 6. Correlogram of Standardized Residuals

Equation: DRU_SE Workfile: DATE_TEZA

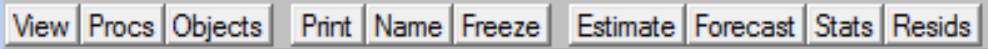

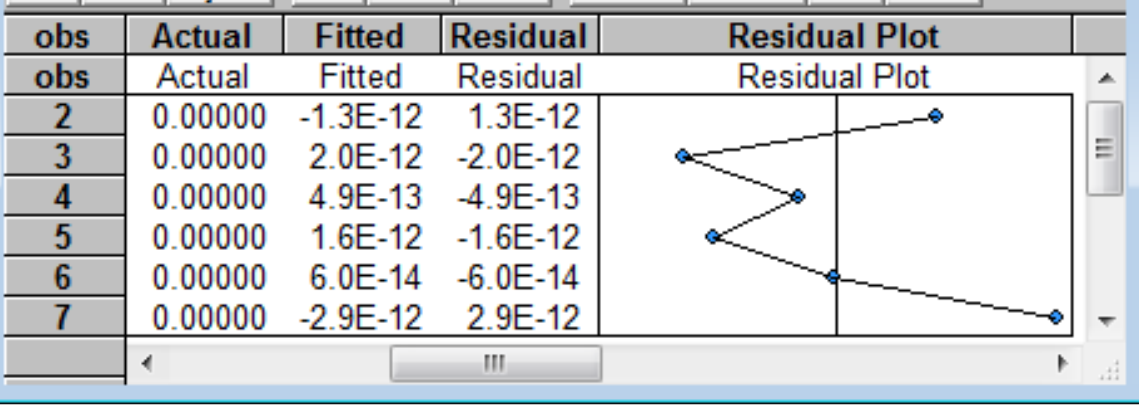


Fig. no. 7. Data for Correlogram of Standardized Residuals

d) West Region

Dependent Variable: Implicit Equation Estimated by GMM

Method: ML - ARCH

Date: 09/11/10 Time: 20:34

Sample(adjusted): 27

Included observations: 6 after adjusting endpoints

Convergence achieved after 1 iterations

LOG(POS_DRU_P_X $(4,1))-\left(\mathrm{C}(1) * \mathrm{LOG}\left(\mathrm{POS} \_D R U \_V \_X(4,1)\right.\right.$

*POP_REG(4,1)*PIB_REG(4,1))+C(2)*LOG(HRD_PRE_V(-1)

*POS_DRU_V_X(4,1)*IRU(4,1)*IRU(4,2)*IRU(4,3)*IRU $(4,4)$

*IRU(4,5)*IRU(4,6)*IRU(4,9)*IRU(4,10)*IRU(4,11)*IRU(4,12))+C(3)

*LOG(SOMAJ_V(-1)*POS_DRU_V_X $(4,1) * \operatorname{IRU}(4,1) * \operatorname{IRU}(4,2)$

$* \operatorname{IRU}(4,3) * \operatorname{IRU}(4,4) * \operatorname{IRU}(4,5) * \operatorname{IRU}(4,6) * \operatorname{IRU}(4,9) * \operatorname{IRU}(4,10) * \operatorname{IRU}(4,11)$

*IRU $(4,12)))$

\begin{tabular}{|c|c|c|c|c|}
\hline & Coefficient & Std. Error & z-Statistic & Prob. \\
\hline$C(1)$ & 0.398389 & 0.001831 & 217.5603 & 0.0000 \\
\hline $\mathrm{C}(2)$ & $-1.69 \mathrm{E}-12$ & $5.94 \mathrm{E}-05$ & $-2.85 \mathrm{E}-08$ & 1.0000 \\
\hline $\mathrm{C}(3)$ & $-2.15 \mathrm{E}-12$ & $6.04 \mathrm{E}-05$ & $-3.55 \mathrm{E}-08$ & 1.0000 \\
\hline $\mathrm{C}(4)$ & $1.37 \mathrm{E}-25$ & $2.01 \mathrm{E}-05$ & $6.81 \mathrm{E}-21$ & 1.0000 \\
\hline $\mathrm{C}(5)$ & 0.150000 & 6.661892 & 0.022516 & 0.9820 \\
\hline $\mathrm{C}(6)$ & 0.600000 & 6.426158 & 0.093368 & 0.9256 \\
\hline Akaike info criterion & -51.87902 & \multirow{3}{*}{\multicolumn{2}{|c|}{$\begin{array}{l}\text { Sum squared resid } \\
\text { Log likelihood }\end{array}$}} & $1.27 \mathrm{E}-24$ \\
\hline Schwarz criterion & -52.08726 & & & 161.6371 \\
\hline Durbin-Watson stat & 1.896105 & & & \\
\hline
\end{tabular}

Equation: DRU_V Workfile: DATE_TEZA

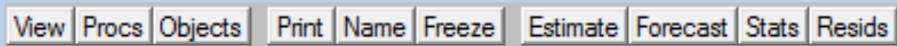

Correlogram of Standardized Residuals

Date: 09/12/10 Time: 00:51

Sample: 27

Included observations: 6

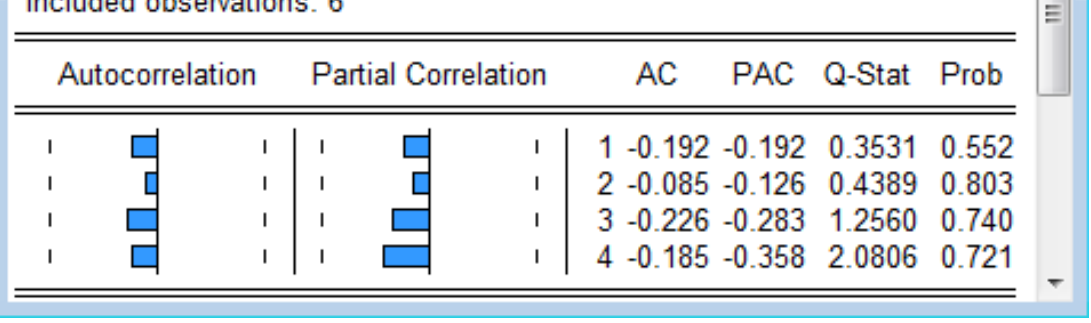

Fig. no. 8. Correlogram of Standardized Residuals 


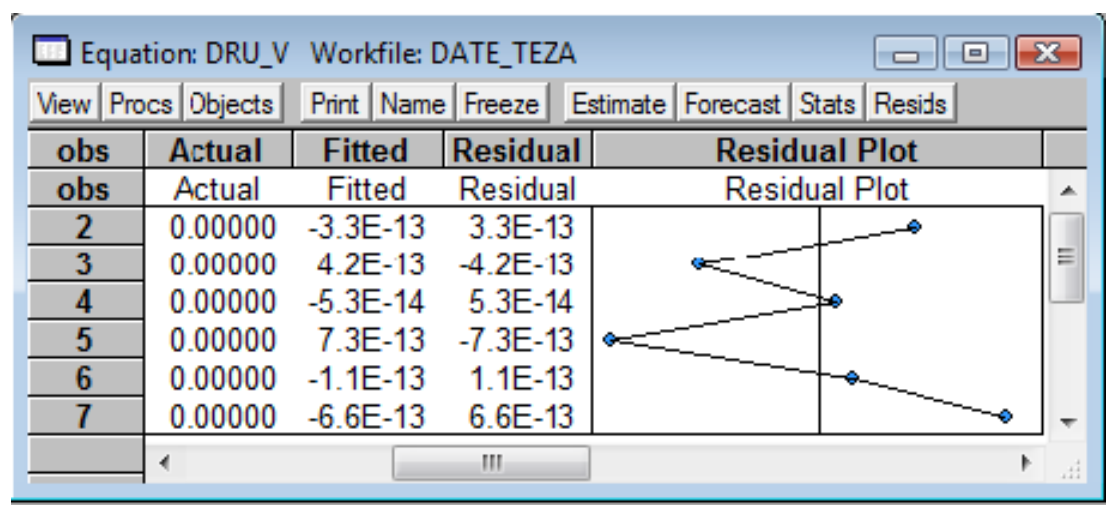

Fig. no. 9. Data for Correlogram of Standardized Residuals

e) North-West Region

Dependent Variable: Implicit Equation Estimated by GMM

Method: ML - ARCH

Date: 09/11/10 Time: 20:35

Sample(adjusted): 27

Included observations: 6 after adjusting endpoints

Convergence achieved after 1 iterations

LOG(POS_DRU_P_X(5,1))-(C(1)*LOG(POS_DRU_V_X $(5,1)$

*POP_REG(5,1)*PIB_REG(5,1))+C(2)*LOG(HRD_PRE_NV(-1)

*POS_DRU_V_X(5,1)*IRU $(5,1) * \operatorname{IRU}(5,2) * \operatorname{IRU}(5,3) * \operatorname{IRU}(5,4)$

$* \operatorname{IRU}(5,5) * \operatorname{IRU}(5,6) * \operatorname{IRU}(5,9) * \operatorname{IRU}(5,10) * \operatorname{IRU}(5,11) * \operatorname{IRU}(5,12))+\mathrm{C}(3)$

*LOG(SOMAJ_NV(-1)*POS_DRU_V_X $(5,1) * \operatorname{IRU}(5,1) * \operatorname{IRU}(5,2)$

$* \operatorname{IRU}(5,3) * \operatorname{IRU}(5,4) * \operatorname{IRU}(5,5) * \operatorname{IRU}(5,6) * \operatorname{IRU}(5,9) * \operatorname{IRU}(5,10) * \operatorname{IRU}(5,11)$

*IRU $(5,12)))$

\begin{tabular}{crrrr}
\hline \hline & Coefficient & Std. Error & z-Statistic & Prob. \\
\hline \hline $\mathrm{C}(1)$ & 0.404949 & 0.002657 & 152.4021 & 0.0000 \\
$\mathrm{C}(2)$ & $-1.53 \mathrm{E}-11$ & $9.71 \mathrm{E}-05$ & $-1.57 \mathrm{E}-07$ & 1.0000 \\
$\mathrm{C}(3)$ & $-4.96 \mathrm{E}-12$ & 0.000119 & $-4.17 \mathrm{E}-08$ & 1.0000 \\
$\mathrm{C}(4)$ & $2.02 \mathrm{E}-24$ & $6.50 \mathrm{E}-06$ & $3.11 \mathrm{E}-19$ & 1.0000 \\
$\mathrm{C}(5)$ & 0.150000 & 7.771543 & 0.019301 & 0.9846 \\
$\mathrm{C}(6)$ & 0.600000 & 9.598346 & 0.062511 & 0.9502 \\
\hline \hline Akaike info criterion & -49.11187 & Sum squared resid & $1.87 \mathrm{E}-23$ \\
Schwarz criterion & -49.32011 & Log likelihood & \\
Durbin-Watson stat & 1.704175 & & \\
\hline \hline
\end{tabular}




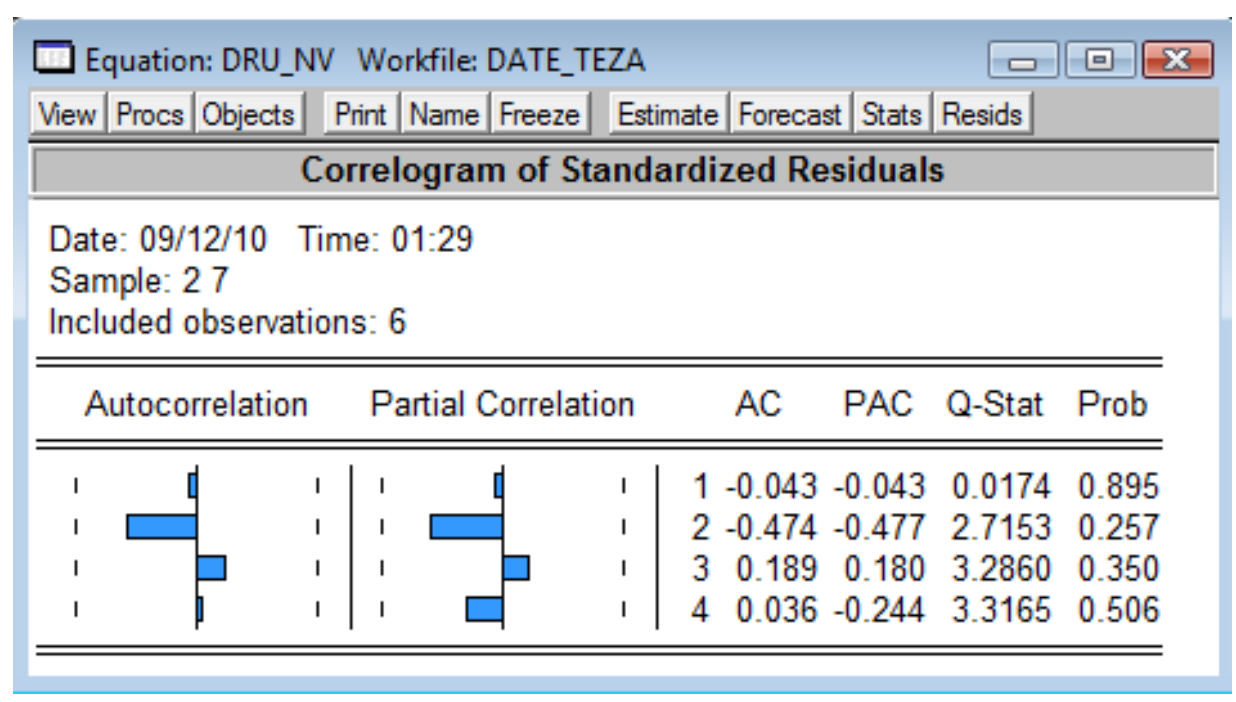

Fig. no. 10. Correlogram of Standardized Residuals

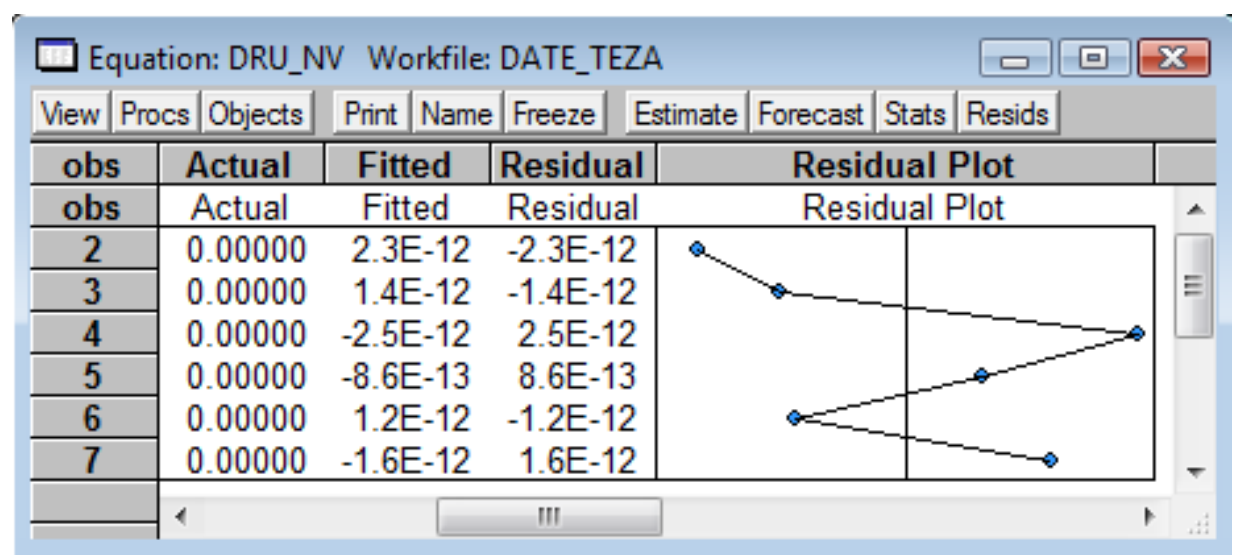

Fig. no. 11. Data for Correlogram of Standardized Residuals

f) North-East Region

Dependent Variable: Implicit Equation Estimated by GMM

Method: ML - ARCH

Date: 09/11/10 Time: 20:43

Sample(adjusted): 27

Included observations: 6 after adjusting endpoints

Convergence achieved after 1 iterations

LOG(POS_DRU_P_X(6,1))-(C(1)*LOG(POS_DRU_V_X $(6,1)$ *POP_REG(6,1)*PIB_REG $(6,1))+\mathrm{C}(2) *$ LOG(HRD_PRE_NE(-1)

*POS_DRU_V_X(6,1)*IRU(6,1)*IRU(6,2)*IRU $(6,3) * \operatorname{IRU}(6,4)$

$* \operatorname{IRU}(6,5) * \operatorname{IRU}(6,6) * \operatorname{IRU}(6,9) * \operatorname{IRU}(6,10) * \operatorname{IRU}(6,11) * \operatorname{IRU}(6,12))+\mathrm{C}(3)$

*LOG(SOMAJ_NE(-1)*POS_DRU_V_X(6,1)*IRU $(6,1) * \operatorname{IRU}(6,2)$

$* \operatorname{IRU}(6,3) * \operatorname{IRU}(6,4) * \operatorname{IRU}(6,5) * \operatorname{IRU}(6,6) * \operatorname{IRU}(6,9) * \operatorname{IRU}(6,10) * \operatorname{IRU}(6,11)$ *IRU $(6,12)))$

\begin{tabular}{rrrrr}
\hline \hline & Coefficient & Std. Error & z-Statistic & Prob. \\
\hline \hline $\mathrm{C}(1)$ & 0.405851 & 0.003239 & 125.3148 & 0.0000 \\
$\mathrm{C}(2)$ & $1.65 \mathrm{E}-11$ & $7.40 \mathrm{E}-05$ & $2.24 \mathrm{E}-07$ & 1.0000
\end{tabular}




\begin{tabular}{crrrr}
$\mathrm{C}(3)$ & $1.96 \mathrm{E}-11$ & $7.86 \mathrm{E}-05$ & $2.50 \mathrm{E}-07$ & 1.0000 \\
$\mathrm{C}(4)$ & $1.99 \mathrm{E}-24$ & $1.61 \mathrm{E}-05$ & $1.24 \mathrm{E}-19$ & 1.0000 \\
$\mathrm{C}(5)$ & 0.150000 & 18.68908 & 0.008026 & 0.9936 \\
$\mathrm{C}(6)$ & 0.600000 & 5.788202 & 0.103659 & 0.9174 \\
\hline \hline Akaike info criterion & -49.32961 & Sum squared resid & $1.84 \mathrm{E}-23$ \\
Schwarz criterion & -49.53785 & Log likelihood & 153.9888 \\
Durbin-Watson stat & 2.727419 & & \\
\hline \hline
\end{tabular}

Equation: DRU_NE Workfile: DATE_TEZA $\square \square \mathrm{x}$

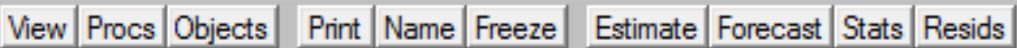
Correlogram of Standardized Residuals

Date: $09 / 12 / 10$ Time: 01:38

Sample: 27

Included observations: 6

\begin{tabular}{cllllllllllll}
\hline \hline \multicolumn{2}{c}{ Autocorrelation } & \multicolumn{5}{c}{ Partial Correlation } & AC & PAC & Q-Stat & Prob \\
\hline \hline & \\
\hline
\end{tabular}

Fig. no. 12. Correlogram of Standardized Residuals

Equation: DRU_NE Workfile: DATE_TEZA

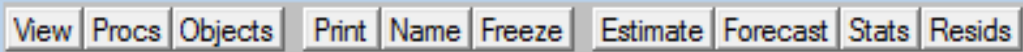

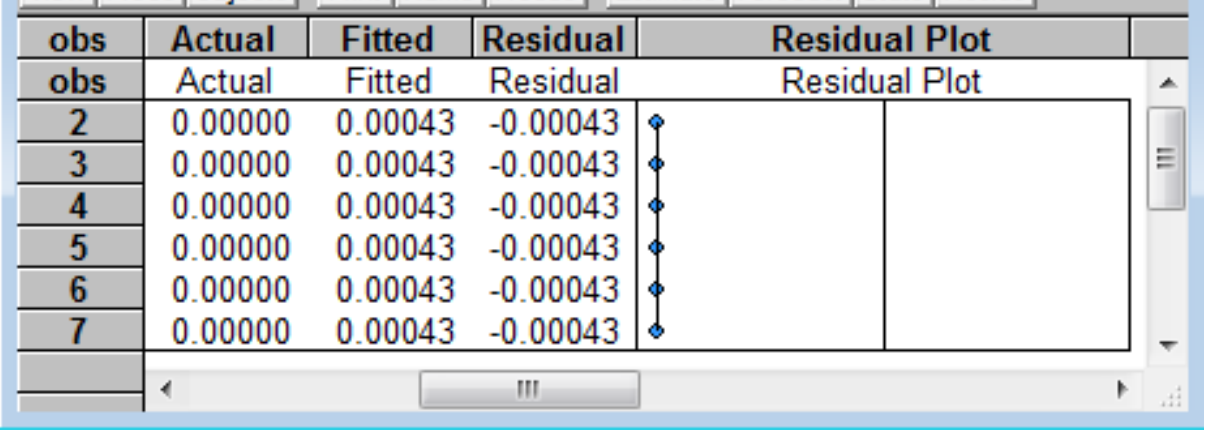

Fig. no. 13. Data for Correlogram of Standardized Residuals

g) Center Region

Dependent Variable: Implicit Equation Estimated by GMM

Method: ML - ARCH

Date: 09/11/10 Time: 20:43

Sample(adjusted): 27

Included observations: 6 after adjusting endpoints

Convergence achieved after 1 iterations

LOG(POS_DRU_P_X(7,1))-(C(1)*LOG(POS_DRU_V_X $(7,1)$

*POP_REG $(7,1) *$ PIB_REG $(7,1))+C(2) *$ LOG(HRD_PRE_C $(-1)$

*POS_DRU_V_X(7,1)*IRU $(7,1) * \operatorname{IRU}(7,2) * \operatorname{IRU}(7, \overline{3}) * \operatorname{IRU}(7,4)$

$* \operatorname{IRU}(7,5) * \operatorname{IRU}(7,6) * \operatorname{IRU}(7,9) * \operatorname{IRU}(7,10) * \operatorname{IRU}(7,11) * \operatorname{IRU}(7,12))+\mathrm{C}(3)$ 
*LOG(SOMAJ_C(-1)*POS_DRU_V_X $(7,1) * \operatorname{IRU}(7,1) * \operatorname{IRU}(7,2)$

$* \operatorname{IRU}(7,3) * \operatorname{IRU}(7,4) * \operatorname{IRU}(7,5) * \operatorname{IRU}(7,6) * \operatorname{IRU}(7,9) * \operatorname{IRU}(7,10) * \operatorname{IRU}(7,11)$

*IRU $(7,12)))$

\begin{tabular}{crrrr}
\hline \hline & Coefficient & Std. Error & z-Statistic & Prob. \\
\hline \hline $\mathrm{C}(1)$ & 0.404277 & 0.035666 & 11.33521 & 0.0000 \\
$\mathrm{C}(2)$ & $5.52 \mathrm{E}-12$ & 0.000129 & $4.28 \mathrm{E}-08$ & 1.0000 \\
$\mathrm{C}(3)$ & $2.72 \mathrm{E}-11$ & 0.000756 & $3.60 \mathrm{E}-08$ & 1.0000 \\
$\mathrm{C}(4)$ & $2.70 \mathrm{E}-24$ & 0.000184 & $1.47 \mathrm{E}-20$ & 1.0000 \\
$\mathrm{C}(5)$ & 0.150000 & 50.10861 & 0.002993 & 0.9976 \\
$\mathrm{C}(6)$ & 0.600000 & 56.57637 & 0.010605 & 0.9915 \\
\hline \hline Akaike info criterion & -48.89349 & Sum squared resid & $2.50 \mathrm{E}-23$ \\
Schwarz criterion & -49.10173 & Log likelihood & 152.6805 \\
Durbin-Watson stat & 1.822623 & & \\
\hline \hline
\end{tabular}

Equation: DRU_C Workfile: DATE_TEZA

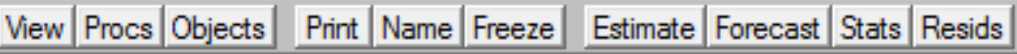

\section{Correlogram of Standardized Residuals}

Date: 09/12/10 Time: 01:48

Sample: 27

Included observations: 6

\begin{tabular}{|c|c|c|c|c|c|c|c|c|c|c|c|c|}
\hline \multicolumn{4}{|c|}{ Autocorrelation } & \multicolumn{4}{|c|}{ Partial Correlation } & \multirow{2}{*}{\multicolumn{2}{|c|}{$\begin{array}{c}A C \\
10.469\end{array}$}} & \multirow{2}{*}{$\begin{array}{l}\text { PAC } \\
0.469\end{array}$} & \multirow{2}{*}{$\frac{\text { Q-Stat }}{2.1145}$} & \multirow{2}{*}{$\frac{\text { Prob }}{0.146}$} \\
\hline I & & 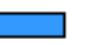 & 1 & I & & & 1 & & & & & \\
\hline 1 & & & 1 & I & $\square$ & & 1 & & 0.033 & -0.241 & 2.1272 & 0.345 \\
\hline I & $\square$ & & 1 & 1 & ᄃ & & 1 & & -0.270 & -0.240 & 3.2945 & 0.348 \\
\hline I & $\square$ & & 1 & 1 & 미 & & 1 & & -0.404 & -0.211 & 7.2032 & 0.126 \\
\hline
\end{tabular}

Fig. no. 14. Correlogram of Standardized Residuals

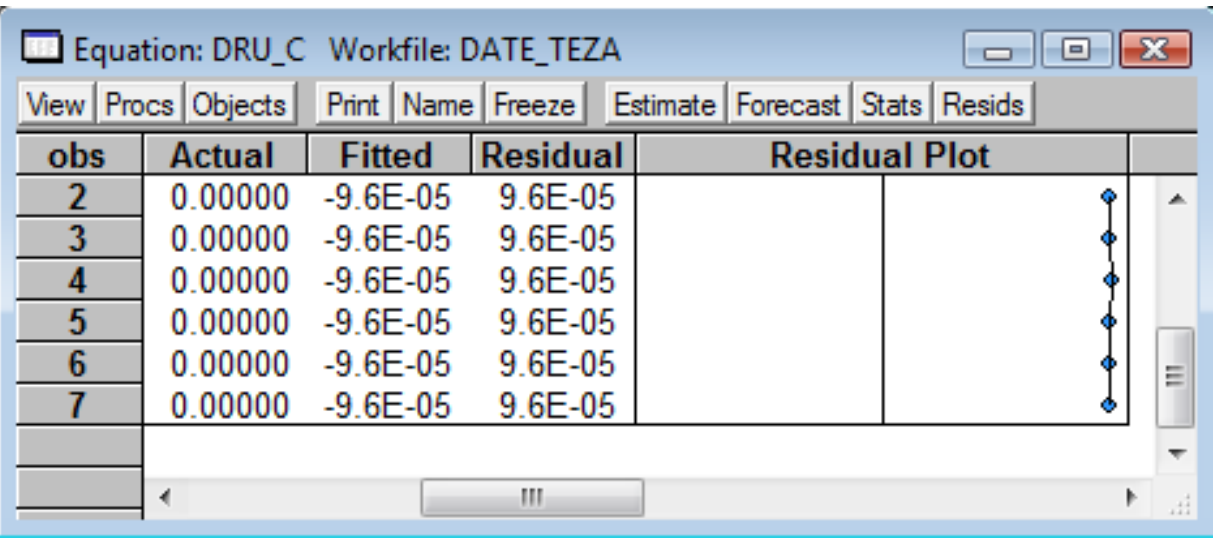

Fig. no. 15. Data for Correlogram of Standardized Residuals

h) Bucharest-Ilfov Region

Dependent Variable: Implicit Equation Estimated by GMM

Method: ML - ARCH

Date: 09/11/10 Time: 20:43

Sample(adjusted): 27 
Included observations: 6 after adjusting endpoints

Convergence achieved after 1 iterations

LOG(POS_DRU_P_X (8,1))-(C(1)*LOG(POS_DRU_V_X $(8,1)$

*POP_REG $(8,1) *$ PIB_REG $(8,1))+\mathrm{C}(2) *$ LOG(HRD_PRE_BIF(-1)

*POS_DRU_V_X $(8,1) * \operatorname{IRU}(8,1) * \operatorname{IRU}(8,2) * \operatorname{IRU}(8,3) * \operatorname{IRU}(8,4)$

$* \operatorname{IRU}(8,5) * \operatorname{IRU}(8,6) * \operatorname{IRU}(8,9) * \operatorname{IRU}(8,10) * \operatorname{IRU}(8,11) * \operatorname{IRU}(8,12))+\mathrm{C}(3)$

*LOG(SOMAJ_BIF(-1)*POS_DRU_V_X(8,1)*IRU $(8,1) * \operatorname{IRU}(8,2)$

$* \operatorname{IRU}(8,3) * \operatorname{IRU}(8,4) * \operatorname{IRU}(8,5) * \operatorname{IRU}(8,6) * \operatorname{IRU}(8,9) * \operatorname{IRU}(8,10) * \operatorname{IRU}(8,11)$ *IRU $(8,12)))$

\begin{tabular}{crrrr}
\hline \hline & Coefficient & Std. Error & z-Statistic & Prob. \\
\hline \hline $\mathrm{C}(1)$ & 0.405034 & 0.015304 & 26.46543 & 0.0000 \\
$\mathrm{C}(2)$ & $2.10 \mathrm{E}-12$ & $4.58 \mathrm{E}-05$ & $4.59 \mathrm{E}-08$ & 1.0000 \\
$\mathrm{C}(3)$ & $1.84 \mathrm{E}-12$ & 0.000208 & $8.82 \mathrm{E}-09$ & 1.0000 \\
$\mathrm{C}(4)$ & $3.20 \mathrm{E}-25$ & 0.000184 & $1.74 \mathrm{E}-21$ & 1.0000 \\
$\mathrm{C}(5)$ & 0.150000 & 78.27955 & 0.001916 & 0.9985 \\
$\mathrm{C}(6)$ & 0.600000 & 66.70175 & 0.008995 & 0.9928 \\
\hline \hline Akaike info criterion & -51.13400 & Sum squared resid & $2.95 \mathrm{E}-24$ \\
Schwarz criterion & -51.34224 & Log likelihood & 159.4020 \\
Durbin-Watson stat & 0.770866 & & \\
\hline \hline
\end{tabular}

Equation: DRU_BIF Workfile: DATE_TEZA

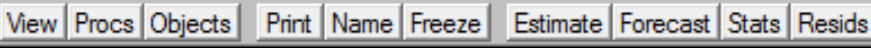

Correlogram of Standardized Residuals

Date: 09/12/10 Time: 01:54

Sample: 27

Included observations: 6

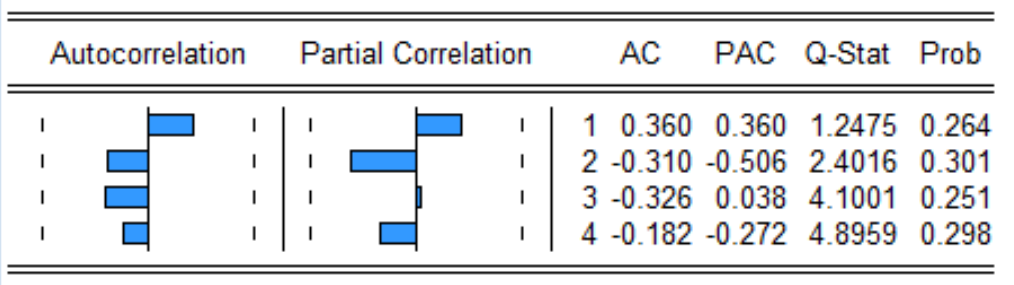

Fig. no. 16. Correlogram of Standardized Residuals

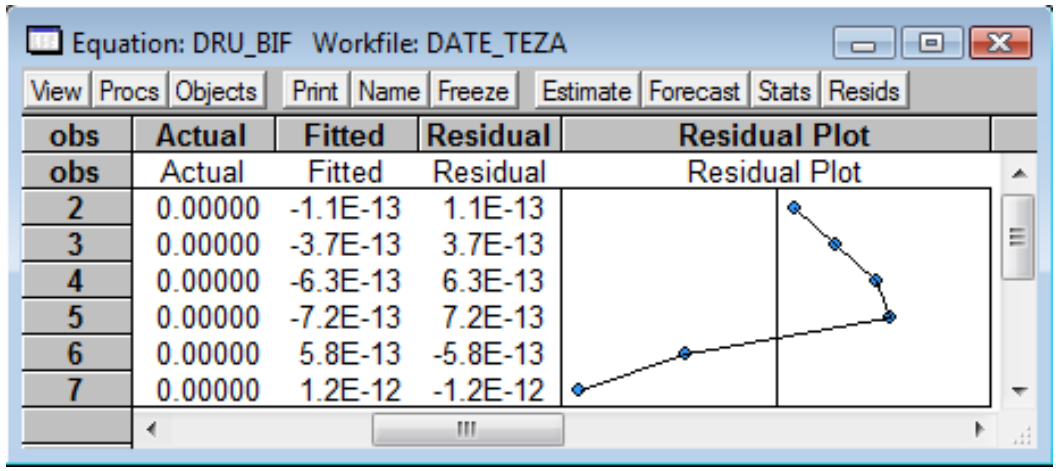

Fig. no. 17. Data for Correlogram of Standardized Residuals

After running the models for each region, the following set of conclusions has been depicted: 
- Due to the differences in magnitude order of several variables it was considered a logarithmic scale in order to facilitate the convergence process. A very peculiar task was to slightly modify the values of time-series in cases when the same value for two consecutive years appeared, hence to eliminate the overflow errors.

- All models converge, but present a quite high degree of volatility. This is explained both by the limited number of observations and by the impossibility of modelling some external factors (e.g. political factors, audit with putting SOP-HRD on standby etc.).

- All applied statistical tests (Akaike, Schwarz, Durbin-Watson) and the corresponding correlograms present normal values and shapes.

- It is very much sensitive to asses the quality of the absorption process at regional level. However, as an example, if using the Akaike criterion, it ranges between -48.59 (South Region) down to -51.87 (West Region). A ranking, under these assumptions, in terms of efficiency of absorption the funds via SOP HRD, is: Region S-C-NW-NE-SE-SW-BIF-W.

$\bigcirc$ The model might be used for future analyses concerning the absorption of structural funds in Romania.

- The model could be refined by introducing supplementary variables and could be also serve as a powerful instrument in developing future strategies for absorbing the structural funds in Romania, to have better programming exercises in the future.

\section{Acknowledgment}

This work was supported by the strategic grant POSDRU/89/1.5/S/61968, Project ID61968 (2009), co-financed by the European Social Fund within the Sectoral Operational Programme Human Resources Development 2007 - 2013.

\section{References}

1. *** Ghiduri de finanţare PHARE Coeziune economică şi Socială 2000-2006.

2. *** Institutul Naţional de Statistică, Anuarele 2000-2009.

3. Bollerslev, T. (1986) Generalized Autoregressive Conditional Heteroskedasticity, Journal of Econometrics, 31, 307-327.

4. Engle, R.F. (1982) Autoregressive Conditional Heteroskedasticity with Estimates of the Variance of U.K. Inflation," Econometrica, 50, 987-1008.

5. Bollerslev T., Chou Y. R, Kroner F. K., (1992) Arch Modeling in Finance: AReview of the Theory and Empirical Evidence, Editura Journal of Econometrics, 52, 5-59.

6. Bradley John, Janusz Zaleski, (2003) Modelling EU accession and Structural Fund impacts using the new Polish HERMIN model - Report.

7. Capello, R. (2007) Regional Economics, Editura Routledge, Marea Britanie.

8. Engle, R.F., Lilien, M., and Robins, P. (1987) Estimating Time Varying Risk Premia in the Term Structure: The ARCH-M Model, Econometrica, 55, 391-407.

9. Gherghinescu, O., Iova, C., Rinderu, P., (2009) Financing human resources development at regional level. Comparison between HRD pre-accession funds and European Social Fund absorption, Metalurgia International, Vol.XIV, Special issue no.13, ISSN: 1582-2214

10. Rinderu, P., (2008) Econometric model for analyzing HRD funds absorption at regional level - North-East region, Annals of the University of Craiova - Economic series.

11. Rinderu, P., Iova, C., Gherghinescu, O., Neagoe-Bacanu, D., (2009) Comparison between HRD pre-accession funds and european social fund absorption at regional level in Romania, 5th International Conference on Applied Business Research, Malta, ISBN: 978-80-7375325-2, www.icabr.com 